\title{
Are There any Predictive Values of Mean Platelet Volume (MPV) and MPV/Platelet Count Ratio in Patients with Spontaneous Abortion?
}

\author{
Pınar Hanife Kara', Seran Ünlüer ${ }^{2}$ \\ 'Department of Emergency Medicine, İzmir Katip Çelebi University, Atatürk Training and Research Hospital, İzmir, Turkey \\ ${ }^{2}$ Department of Emergency Medicine, Buca Seyfi Demirsoy State Hospital, İzmir, Turkey
}

\begin{abstract}
Aim: The objective of this study was to evaluate the predictive value of mean platelet volume (MPV) and MPV/platelet count ratio and to determine whether they are based on hemoconcentration in patients with spontaneous abortions.

Materials and Methods: A total of 271 women with singleton pregnancies between January 1, 2012, and June 30, 2012, were included (171 healthy pregnant, 200 spontaneous abortion). Study data were obtained from hospital data processing system, emergency department charts, and consultation records. Mann-Whitney U, Kruskal-Wallis $\mathrm{H}$ (post-hoc Bonferroni-corrected Mann-Whitney U), and logistic regression (LR forward method) analyses were used as appropriate.

Results: Among the patients with MPV $>9.1 \mathrm{fl}$, it was observed that they had a greater rate of spontaneous abortions with vaginal bleeding. The levels of hemoglobin, hematocrit, urea, and creatinine as well as platelets count were significantly higher in women with a spontaneous abortion ( $p<0.05)$. There were no significant differences between abortion subgroups with bleeding and non-bleeding symptoms $(p>0.05)$.

Conclusion: These results suggest that minimal changes in both MPV and MPV/platelet count ratio can aid the clinician during routine pregnancy visits or in the emergency department by serving as indicators of an increased risk of abortion, but more importantly the elevated hemoglobin despite bleeding strongly suggests hemoconcentration possibility.
\end{abstract}

Keywords: Emergency, pregnancy, routine laboratory, spontaneous abortion

\section{Introduction}

Pregnancy is a normal physiological phenomenon with many biochemical changes. Nevertheless, early pregnancy loss is a common complication of pregnancy, with an incidence of 50\%-70\% (13 ). The World Health Organization (WHO) has defined spontaneous abortion as the loss of a fetus before 20 weeks or that is weighing less than 500 grams (4). More than $60 \%$ of spontaneous abortions are due to maternal or paternal chromosomal abnormalities and $15 \%$ are due to maternal trauma, infections, dietary deficiencies, diabetes mellitus, hypothyroidism, lupus anticoagulant-anticardiolipin-antiphospholipid antibody syndrome, advanced maternal age, toxic effects, or anatomic disorders. Nevertheless, one quarter of cases have no clear cause (4). Blood tests are routinely used to identify metabolic abnormalities that reflect conditions harmful to the mother or fetus, but routine laboratory reference values may not reflect natural alterations of pregnancy. There are few reports of first and second trimester normal reference intervals for pregnant women $(2,5,6)$. Indeed, biochemical changes associated with pregnancy that assist the development and survival of the fetus can alter laboratory values so they fall outside the normal, non-pregnant range and are thus misinterpreted as abnormal (1). It is critical to characterize the normal and abnormal changes of pregnancy, as laboratory results are often used to influence management of both the mother and the child (1). Without appropriate reference ranges, there is both an increased risk of missing important changes due to pathological conditions and a risk of erroneously interpreting normal changes as pathological events (5).

Many previous studies have identified normal pregnancy laboratory reference values, determined pregnancy-related changes in organ function, and studied special biomarkers, but no studies have heretofore compared laboratory values in women with spontaneous

Correspondence to: Pınar Hanife Kara e-mail: hpinarkara@hotmail.com 
abortion to values in healthy pregnant women $(2,6)$. Comparing laboratory results of women with a spontaneous abortion to results of normal pregnant women at the same gestational age may more clearly identify laboratory risk factors for abortion than comparing them to the normal reference values of non-pregnant women. However, there is no predictive factor to estimate spontaneous abortions yet.

The objective of this study was to evaluate the predictive value of mean platelet volume (MPV) and MPV/platelet count ratio and to determine whether they are based on hemoconcentration in patients with spontaneous abortions.

\section{Materials and Methods}

This study was approved by our Local Research Ethics Committee (No. 9). Of the 2738 women examined in the Ataturk Research and Training Hospital Emergency Department of Katip Celebi University (Izmir, Turkey) between January 01, 2012, and June 30, 2012, data were retrospectively collected from 171 normal, healthy pregnant women in the first 20 weeks of gestation and 200 women with spontaneous abortion. Only women with singleton pregnancies were included.

Normal pregnancy was defined as a pregnancy with no evidence of maternal or fetal disease, in which the mother did not take medications or exhibit abnormal vaginal bleeding or other pregnancy-related complications throughout the pregnancy. Additional inclusion criteria for the healthy pregnancy group were a normal outcome of the pregnancy and all follow-up conducted at our hospital. Only women with totally uncomplicated pregnancies were included in this group to ensure that the results were not influenced by any pathological condition. The abortion group included women in whom a gynecologist/obstetrician diagnosed imminent abortion, abortion incipience, incomplete abortion, complete abortion, missed abortion, or septic abortion. Gestational age was estimated by the crown--rump length, as determined by an ultrasound scan performed by the attending obstetrician (Sonoace X8, Medison Inc., Seoul, Korea). In cases with no signs of conception, the gestational age was determined according to the date of the last menstrual period.

Obstetrical history, physical examination, and other clinical data were obtained from the emergency department charts and obstetrics consultation records.

Laboratory variables used were: levels of hemoglobin $(\mathrm{Hb}, \mathrm{g} / \mathrm{fL})$, hematocrit $(\mathrm{Hct}, \%)$, urea $(\mathrm{mg} / \mathrm{dL})$, and creatinine $(\mathrm{mg} / \mathrm{dL})$ as well as counts of white blood cells (WBC, K/mL) and platelets (PIt, K/mL) in addition to MPV (fl) and MPV/platelet counts ratio.
All blood laboratory samples were analysed for complete blood count, routine biochemistry, and basic coagulation tests in the same laboratory. Levels of $\mathrm{Hb}$ and $\mathrm{Hct}$ and counts of WBC and Plt as well as MPV testing were performed using the Automated Hematology Analyser (Cell-Dyn 3700 Abbott, Abbott Park, IL). Biochemical tests including urea and creatinine levels were conducted with an autoanalyzer (C16000; Abbott).

\section{Statistical analysis}

Statistical analysis was performed using the Statistical Package for the Social Sciences (SPSS Inc.; Chicago, IL, USA) version 15.0for Windows program, with $95 \%$ confidence intervals. The distribution of the data was tested by applying the Kolmogorov-Smirnov and Shapiro-Wilk tests of normality. The variables failed to show a normal distribution $(p<0.05)$; therefore, non-parametric tests were used. The Mann-Whitney $U$ test was used for the comparison of two groups of continuous data. The Kruskal-Wallis $\mathrm{H}$ (post-hoc Bonferroni-corrected Mann-Whitney $U$ ) test was used to compare more than two groups, the results of one-way analysis of variance comparing all laboratory blood test parameters between abortion subgroups are shown. Otherwise the cut-off value of the MPV was calculated with receiver operator characteristics (ROC) curve analyses. The standard deviation and 95\% confidence intervals for the mean were determined for all laboratory parameters. Logistic regression (LR forward method) analyses were performed to determine the effect of variables on the occurrence of a spontaneous abortion. Statistical significance was defined as $\mathrm{p}<0.05$.

\section{Results}

This study included 371 women: 200 in the abortion group and 171 in the healthy pregnancy group. The diagnoses for the abortion cases were distributed as follows: abortion imminence, $36 \%(n=72)$; missed abortion, $23.5 \%(n=47)$; incomplete abortion, $27.5 \%(n=55)$; complete abortion, 7.5\% ( $n=15)$; and abortion incipience, $5.5 \%$ $(n=11)$. The abortion group was also divided into bleeding $(n=179$, $89.5 \%)$ and non-bleeding $(n=21,10.5 \%)$ subgroups, based on their symptoms upon arrival in the emergency room.

The patients' baseline characteristics are listed in Table 1. Mean age, number of pregnancies, number of births, and number of previous abortions were significantly higher in the abortion group than in the healthy pregnancy group $(p<0.05)$, and the gestational age of the abortion group was significantly lower than the healthy pregnancy group $(p<0.001)$. Post-hoc Bonferroni-corrected Mann-Whitney $U$

Table 1. Patient characteristics: age, number of pregnancies, number of births, and number of previous abortions

\begin{tabular}{|c|c|c|c|c|c|c|c|c|c|c|}
\hline & $\mathbf{A b c}$ & tion $\mathbf{g}$ & & Healthy p & egnan & group & & Total & & \\
\hline & Mean \pm SD & Min & Max & Mean \pm SD & Min & Max & Mean \pm SD & Min & Max & $\mathbf{p}^{*}$ \\
\hline Age (y) & $28.76 \pm 6.52$ & 17 & 46 & $25.5 \pm 5.5$ & 16 & 42 & $27.25 \pm 6.28$ & 16 & 46 & $<0.001$ \\
\hline Gestational age (days) & $66.59 \pm 25.89$ & 20 & 147 & $81.21 \pm 33.35$ & 14 & 144 & $73.27 \pm 30.38$ & 14 & 147 & $<0.001$ \\
\hline Number of pregnancies & $2.48 \pm 1.6$ & 1 & 9 & $2.01 \pm 1.3$ & 1 & 7 & $2.26 \pm 1.48$ & 1 & 9 & 0.002 \\
\hline Number of births & $1.05 \pm 1.25$ & 0 & 8 & $0.78 \pm 1.03$ & 0 & 4 & $0.93 \pm 1.16$ & 0 & 8 & 0.031 \\
\hline Number of prior abortions & $0.46 \pm 0.96$ & 0 & 8 & $0.22 \pm 0.53$ & 0 & 3 & $0.35 \pm 0.8$ & 0 & 8 & 0.003 \\
\hline
\end{tabular}


Table 2. Distribution of blood laboratory values of the abortion and healthy pregnancy groups

\begin{tabular}{|l|c|c|c|c|c|}
\hline \multirow{2}{*}{} & \multicolumn{2}{|c|}{$\begin{array}{c}\text { Abortion } \\
\text { group }\end{array}$} & \multicolumn{2}{c|}{$\begin{array}{c}\text { Healthy pregnancy } \\
\text { group }\end{array}$} & \multirow{2}{*}{ ( } \\
\cline { 2 - 5 } & Mean & SD & Mean & SD & p* \\
\hline $\mathrm{WBC}(\mathrm{K} / \mu \mathrm{L})$ & 10.06 & 3.17 & 9.35 & 1.71 & 0.282 \\
\hline $\mathrm{Hb}(\mathrm{g} / \mathrm{dL})$ & 12.39 & 1.24 & 11.94 & 1.01 & $<0.001$ \\
\hline $\mathrm{Hct}(\%)$ & 35.85 & 3.19 & 35.31 & 12.03 & $<0.001$ \\
\hline $\mathrm{Plt}(\mathrm{K} / \mu \mathrm{L})$ & 271.44 & 64.27 & 247.72 & 55.41 & $<0.001$ \\
\hline $\mathrm{MPV}(\mathrm{fL})$ & 8.60 & 1.43 & 8.39 & 1.36 & 0.433 \\
\hline $\begin{array}{l}\text { MPV/platelets } \\
\text { count ratio }\end{array}$ & 0.03 & 0.00011 & 0.04 & 0.00101 & $<0.001$ \\
\hline Urea (mg/dL) & 8.98 & 2.85 & 8.36 & 2.44 & 0.039 \\
\hline Creatinine (mg/dL) & 0.59 & 0.07 & 0.56 & 0.07 & $<0.001$ \\
\hline
\end{tabular}

*Mann-Whitney U test. SD: standard deviation; WBC: white blood cell; $\mathrm{Hb}$ : hemoglobin; Hct: hematocrit; Plt: platelet; MPV: mean platelet volume

Table 3. Distribution of blood laboratory values of abortion cases based on their bleeding status at emergency room arrival

\begin{tabular}{|l|c|c|c|}
\hline \multirow{2}{*}{} & $\begin{array}{c}\text { Bleeding } \\
\text { (n=179) }\end{array}$ & $\begin{array}{c}\text { Non-bleeding } \\
\text { (n=21) }\end{array}$ & \multirow{2}{*}{ ' } \\
\cline { 2 - 3 } & Mean \pm SD & Mean \pm SD & \multirow{2}{*}{ * } \\
\hline WBC (K/L) & $10.12 \pm 3.23$ & $9.41 \pm 2.65$ & 0.480 \\
\hline Hb (g/dL) & $12.34 \pm 1.28$ & $12.89 \pm 0.75$ & 0.093 \\
\hline Plt (K/L) & $35.73 \pm 3.28$ & $37.02 \pm 1.92$ & 0.096 \\
\hline MPV (fL) & $269.56 \pm 64.32$ & $287.75 \pm 64.71$ & 0.193 \\
\hline MPV/platelets count ratio & $0.03 \pm 0.00024$ & $0.03 \pm 0.00018$ & $<0.001$ \\
\hline Urea (mg/dL) & $9.13 \pm 2.91$ & $7.50 \pm 1.64$ & 0.007 \\
\hline Creatinine (mg/dL) & $0.59 \pm 0.07$ & $0.56 \pm 0.06$ & 0.133 \\
\hline
\end{tabular}

*Mann-Whitney U test. SD: standard deviation; WBC: white blood cell; Hb: hemoglobin; Hct: hematocrit; Plt: platelet; MPV: mean platelet volume tests of baseline characteristics for the abortion subgroups revealed no significant differences between subgroups for mean age, gestational age, number of pregnancies, and number of previous abortions ( $p>0.05$ ), although the number of births was significantly higher in the incomplete abortion group than in the complete abortion group $(\mathrm{p}<0.05)$.

As noted in Table 2, Hb, Hct, Plt, urea, and creatinine values were significantly higher in the abortion group than in the healthy pregnancy group $(p<0.05)$. However, the MPV values were not significantly different between the two groups ( $p>0.05)$. Otherwise, the MPV/ platelet count ratio levels were significantly lower in the abortion group than in control group $(\mathrm{p}<0.05)$.

The laboratory results of the bleeding and non-bleeding groups were compared with the Mann-Whitney $U$ test to help understand the association between bleeding and the abortion process (Table 3). Urea levels were significantly higher in the bleeding group but MPV/ platelet count ratio was significantly lower in this group $(p<0.05$ and $p<0.05$, respectively).

Cut-off value for MPV was taken as $9.1 \mathrm{fL}$, and the patients were divided in two groups. According to this classification, patients with MPV value higher than $9.1 \mathrm{fL}$ have suffered from spontaneous abortion with bleeding ( $p>0.05)$. Moreover, it was defined a positive and significant correlation between higher levels than $9.1 \mathrm{fL}$ of the MPV and increased urea $(r=0.621, p=0.031)$, creatinine $(r=0.539, p=0.037)$, $\mathrm{Hb}(r=0.648, p=0.018)$, Hct $(r=0.496, p=0.042)$ levels, decreased MPV/ platelets count ratio $(r=0.598, p=0.029)$, and spontaneous abortions with bleeding $(r=0.618, p=0.024)$.

According to the cut-off level of MPV, we found that MPV levels higher than $9.1 \mathrm{fL}$ had been seen a $85 \%$ specificity, $91 \%$ sensitivity, $94 \%$ accuracy, $61 \%$ negative likelihood value, and $74 \%$ positive likelihood value to predict to patients who had spontaneous abortion with bleeding.

The results of one-way analysis of variance comparing all laboratory blood test parameters between abortion subgroups are shown in Table 4.

LR analysis with a forward LR method identified parameters that affected the occurrence of abortion (Table 5).

Table 4. Distribution of blood laboratory values of the abortion subgroups

\begin{tabular}{|c|c|c|c|c|c|c|}
\hline & & & Diagnosis & & & \\
\hline & $\begin{array}{c}\text { Abortion } \\
\text { imminence }\end{array}$ & $\begin{array}{c}\text { Missed } \\
\text { abortion }\end{array}$ & $\begin{array}{c}\text { Incomplete } \\
\text { abortion }\end{array}$ & $\begin{array}{l}\text { Complete } \\
\text { abortion }\end{array}$ & $\begin{array}{c}\text { Abortion } \\
\text { incipience }\end{array}$ & \\
\hline & Mean \pm SD & Mean \pm SD & Mean \pm SD & Mean \pm SD & Mean \pm SD & $\mathbf{p}^{*}$ \\
\hline WBC (K/L) & $9.8 \pm 3.44$ & $10.31 \pm 3.07$ & $10.24 \pm 3.31$ & $9.8 \pm 2.39$ & $10.11 \pm 2.10$ & 0.462 \\
\hline $\mathrm{Hb}(\mathrm{g} / \mathrm{dL})$ & $12.27 \pm 1.35$ & $12.48 \pm 1.29$ & $12.41 \pm 1.19$ & $12.42 \pm 0.94$ & $12.58 \pm 1.05$ & 0.824 \\
\hline Hct (\%) & $35.67 \pm 3.35$ & $36.05 \pm 3.61$ & $35.93 \pm 2.84$ & $35.91 \pm 2.77$ & $35.71 \pm 2.86$ & 0.927 \\
\hline Plt (K/L) & $261.98 \pm 65.67$ & $282.34 \pm 65.26$ & $276.38 \pm 63.64$ & $270.47 \pm 62.79$ & $263.36 \pm 56.46$ & 0.493 \\
\hline MPV (fL) & $8.59 \pm 1.69$ & $8.62 \pm 1.36$ & $8.38 \pm 1.13$ & $8.54 \pm 1.11$ & $9.7 \pm 1.34$ & 0.071 \\
\hline MPV/platelets count ratio & $0.03 \pm 0.00012$ & $0.03 \pm 0.00023$ & $0.03 \pm 0.00019$ & $0.03 \pm 0.00016$ & $0.03 \pm 0.00019$ & 0.068 \\
\hline Urea $(\mathrm{mg} / \mathrm{dL})$ & $9.06 \pm 3.34$ & $9.33 \pm 2.49$ & $8.80 \pm 2.82$ & $9.27 \pm 1.83$ & $7.64 \pm 1.69$ & 0.266 \\
\hline Creatinine $(\mathrm{mg} / \mathrm{dL})$ & $0.59 \pm 0.07$ & $0.59 \pm 0.07$ & $0.59 \pm 0.08$ & $0.62 \pm 0.08$ & $0.59 \pm 0.07$ & 0.744 \\
\hline
\end{tabular}


Table 5. LR analysis (forward LR) of variables associated with the risk of an abortion

\begin{tabular}{|l|c|c|c|c|c|c|c|c|}
\hline Variable & B & Standard error & Wald & df & Significance & Exp(B) * & \multicolumn{2}{|c|}{$95 \%$ C.I. for Exp(B) } \\
\hline Maternal age & 0.103 & 0.024 & 18.813 & 1 & $<0.001$ & 1.108 & 1.058 & 1.161 \\
\hline Gestational age & -0.014 & 0.005 & 7.318 & 1 & 0.007 & 0.986 & 0.977 & 0.996 \\
\hline WBC & 0.157 & 0.061 & 6.681 & 1 & 0.010 & 1.170 & 1.039 & 1.318 \\
\hline Hb & 0.316 & 0.134 & 5.523 & 1 & 0.019 & 1.372 & 1.054 & 1.785 \\
\hline Plt & 0.006 & 0.002 & 6.641 & 1 & 0.010 & 1.006 & 1.002 & 1.011 \\
\hline MPV/platelets count ratio & 0.008 & 0.002 & 6.514 & 1 & 0.000 & 1.011 & 1.004 & 1.018 \\
\hline Constant & -39.927 & 7.913 & 25.459 & 1 & 0.000 & 0.000 & \\
\hline
\end{tabular}

\section{Discussion}

Blood tests were often recommended during pregnancy. The laboratory reference intervals were usually based on the results of tests obtained in healthy or non-pregnant women. This is not optimal, as many biological markers change during pregnancy, and using the most appropriate reference values is necessary to make correct clinical decisions (5-8).

During normal pregnancy, an increased cardiac output was found to improve renal perfusion, which led to an increased glomerular filtration rate (GFR), compared to pre-pregnancy values. The increase in GFR was observable within 1 month of conception, peaked at approximately $40 \%-50 \%$ above baseline by the early second trimester, and then declined slightly near the end of pregnancy (9). Increased GFR led to lower serum creatinine and urea levels in pregnant women $(5,10,11)$. Indeed, even in the presence of severe proteinuria, serum urea, and creatinine usually remained within the normal range during pregnancy $(12,13)$. Our findings that the urea and creatinine levels were statistically higher in the abortion group than in the healthy pregnancy group suggest that renal adaptation is less pronounced in women undergoing spontaneous abortion than in healthy pregnant women.

The WHO defines anemia in pregnant women as an $\mathrm{Hb}<110 \mathrm{~g} / \mathrm{L}$ or a $\mathrm{Hct}<6.83 \mathrm{mmol} / \mathrm{L}$ or $<33 \%$ (9). Neither of the study groups was anemic according to this definition, but significantly higher $\mathrm{Hb}, \mathrm{Hct}$, and Plt values were found in the abortion group. These findings were noted, despite the observation that almost $90 \%$ of the abortion group presented with bleeding symptoms. This suggests that hemoconcentration may have been present in the abortion group. Maternal hemoconcentration and high viscosity have been previously reported during severe pre-eclampsia; they may further impede the uteroplacental blood flow that is already compromised by pre-eclampsia, thereby contributing to placental infarction and growth retardation or fetal death $(14,15)$. Blood viscosity reaches a level that impairs microcirculation, and an inadequate amount of oxygen is transported to tissues, similar to the situation with severe anemia (16). Contracted plasma volume resulted in an appearance of greater red cell volume. The principal mechanism for perinatal morbidity and mortality due to pre-eclampsia-such as pregnancy-induced hypertension-is poor placental and fetal perfusion (16).

In normal pregnancy, Plt counts are similar to or slightly lower than those observed in healthy subjects (17). The Plt and Pct levels were higher in our abortion group, but the MPV values were not statistically different between the abortion and healthy pregnancy groups, and there were no statistically significant differences between the abortion subgroups. In contrast, Kosus et al. (2) reported higher MPV values in patients with a missed abortion than in those with a healthy pregnancy. Larger platelets, reflecting higher MPV values, are hemostatically more reactive than smaller platelets and thus have a greater propensity to form thromboses (18). These modifications of the coagulation system result from hormonal changes and are part of a complex physiological adaptation of the organism to pregnancy (19). However, the constant MPV, reflecting the presence of less active platelets, may represent a pre-disseminated intravascular coagulation state or activation of the fibrinolytic system as part of the spontaneous abortion process (20).

Strengths of this study include its blinded collection and interpretation of the data, as well as its large sample size, which give our results narrow confidence intervals. Furthermore, we compared the abortion group with a healthy pregnancy group, whose similar physiological baseline provided a more appropriate comparison than a non-pregnant population. Although there were statistically significant differences in gestational age between patients in the abortion and healthy pregnancy groups, this was likely of no clinical relevance, as the difference between groups was only a few days, and patients in both groups were in the same trimester.

\section{Study limitations}

A limitation of the study became evident when we noted that the $\mathrm{Hb}$ and $\mathrm{Hct}$ levels were elevated in the abortion group despite the presence of vaginal bleeding. Although these elevations suggest the presence of hemoconcentration in the abortion group, it is not possible to determine whether hemoconcentration was the result or cause of the abortion because we were unable to compare the abortion group laboratory values to those obtained from the same patients prior to their abortion. Further studies would be required to more fully address this issue.

\section{Conclusion}

Laboratory parameters of healthy pregnant women should reflect the physiological changes during pregnancy. Our results demonstrate small, although significant, differences in MPV level and MPV/platelet ratio as well as some variables probably related with hemoconcentration such as $\mathrm{Hb}$, Hct, urea, creatinine levels, and Plt count between the abortion and healthy pregnancy groups. These results may assist clini- 
cians, as the observed changes in routine parameters during routine pregnancy visits may identify risk factors for abortion. Although there are various statistical differences in the results of our study, they are so small and within the error range of the laboratory measurements. They probably have no practical significance, but the most important point in these results has been indicated that there is a marked finding about hemoconcentration according these results. Moreover, this situation can be associated with poor fetal outcome, and it can be an avoidable subject especially for imminent abortion. Although statistically significant differences were noted, further studies are required to identify clinically useful cutoffs for these parameters.

Ethics Committee Approval: This study was approved by the Katip Celebi University Ataturk Research and Training Hospital Research Ethics Committee (date of approval: 04.01.2013, No. 9).

Informed Consent: There is no need for informed consent because it is a retrospective data study.

Peer-review: Externally peer-reviewed.

Conflict of Interest: No conflict of interest was declared by the authors.

Financial Disclosure: The authors declared that this study has received no financial support.

\section{References}

1. Tran HA. Biochemical tests for abnormalities in pregnancy. Aust Prescr 2006; 29: 48-52. [CrossRef]

2. Kosus N, Kosus A, Yildirim M, Duran M, Turhan NO. Mean platelet volume as a marker of thrombosis in patients with missed abortion. Acta Haematol 2011; 125: 208-9. [CrossRef]

3. Jauniaux E, Burton GJ. Pathophysiology of histological changes in early pregnancy loss. Placenta 2005; 26: 114-23. [CrossRef]

4. Judith E. Tintinalli, (Editor in Chief). Tintinalli's Emergency Medicine A Comprehensive Study Guide 7th Edition. Mc Graw Hill. 2011. ISBN 9780 07 174466. Ectopic Pregnancy and Emergencies in the first 20 weeks of Pregnancy. Richard S. Krause, David M. Janicke, Rita K. Cydulka. p: 676-84.

5. Larsson A, Palm M, Hansson LO, Axelsson O. Reference values for clinical chemistry tests during normal pregnancy. BJOG 2008; 115: 874-81. [CrossRef]
6. Edelstam G, Löwbeer C, Kral G, Gustafsson SA, Venge P. New reference values for routine blood samples and human neutrophilic lipocalin during third-trimester pregnancy. Scand J Clin Lab Invest 2001; 61: 58392. [CrossRef]

7. Torgersen KL, Curran CA. A systematic approach to the physiologic adaptations of pregnancy. Crit Care Nurs Q 2006; 29: 2-19. [CrossRef]

8. Klajnbard A, Szecsi PB, Colov NP, Andersen MR, Jørgensen M, Bjørngaard $B$, et al. Laboratory reference intervals during pregnancy, delivery and the early postpartum period. Clin Chem Lab Med 2010; 48: 237-48. [CrossRef]

9. Davidson JM, Dunlop W. Renal hemodynamics and tubular function in normal human pregnancy. Kidney Int 1980; 18: 152-61. [CrossRef]

10. Giovanni L, Maria LG, Mauro R, Carlotta M, Federica R, Fabrizio P, et al. Thrombophilia and damage of kidney during pregnancy. J Prenat Med 2011; 5: 78-82.

11. Ganesan C, Maynard SE. Acute kidney injury in pregnancy: the thrombotic microangiopathies. J Nephrol 2011; 24: 554-63. [CrossRef]

12. Indumati V, Kodliwadmath MV, Sheela MK. The role of serum electrolytes in pregnancy induced hypertension. Journal of Clinical and Diagnostic Research 2011; 5: 66-9.

13. Maynard SE, Thadhani R. Pregnancy and the kidney. J Am Soc Nephrol 2009; 20: 14-22. [CrossRef]

14. Sagen N, Koller O, Haram K. Haemoconcentration in severe pre-eclampsia. Br J Obstet Gynaecol 1982; 89: 802-5. [CrossRef]

15. Koller O, Sandvei R, Sagen N. High hemoglobin levels during pregnancy and fetal risk. Int J Gynaecol Obstet 1980; 18: 53-6. [CrossRef]

16. Yip R. Significance of an abnormally low or high hemoglobin concentration during pregnancy: special consideration of iron nutrition. Am J Clin Nutr 2000; 72: 272-9.

17. Boehlen F, Hohlfeld P, Extermann P, Perneger TV, de Moerloose P. Platelet count at term pregnancy: a reappraisal of the threshold. Obstet Gynecol 2000; 95: 29-33. [CrossRef]

18. Martin JF, Trowbridge EA, Salmon G, Plumb J. The biological significance of platelet volume: its relationship to bleeding time, platelet thromboxane B2 production and megakaryocyte nuclear DNA concentration. Thromb Res 1983; 32: 443-60. [CrossRef]

19. Uchikova $E H$, Ledjev II. Changes in haemostasis during normal pregnancy. Eur J Obstet Gynecol Reprod Biol 2005; 119: 185-8. [CrossRef]

20. Cerneca F, Ricci G, Simeone R, Malisano M, Alberico S, Guaschino S. Coagulation and fibrinolysis changes in normal pregnancy. Increased levels of procoagulants and reduced levels of inhibitors during pregnancy induce a hypercoagulable state, combined with a reactive fibrinolysis. Eur $\mathrm{J} \mathrm{Ob-}$ stet Gynecol Reprod Biol 1997; 73: 31-6. [CrossRef] 